\title{
Vectores éticos de innovación oculta en la tecnología social*
} Ethical Vectors of Hidden Innovation in Social Technology

JaVIER Bustamante Donas

Universidad Complutense de Madrid

RESUMEN. En este artículo se estudia la conexión entre tecnología social e innovación oculta a partir de un conjunto de vectores éti$\cos$. Estos vectores éticos permiten que aflore y se difunda la innovación oculta que se produce a través de la tecnología social en entornos colaborativos. Entender la dimensión ética de la tecnología social permite identificar el papel que juega un conjunto de leyes (ley de Metcalfe, ley de rendimientos crecientes de adopción y ley de externalidades positivas) en el desarrollo de prácticas de empoderamiento social en dichos entornos, definidos como "comunidades de conocimiento". Se analizan estos vectores éticos presentes en la tecnología social a partir de las siguientes categorías: conocimiento e innovación comunitarios, participación ciudadana y democracia, diálogo entre saberes populares y saberes científicos, y proceso pedagógico holístico.

Palabras clave: tecnología social, innovación oculta, comunidades de conocimiento, ética y tecnología.
ABSTRACT. This article studies the connection between social technology and hidden innovation from a set of ethical vectors. These ethical vectors foster the rising and spreading of a hidden innovation created in collaborative environments by means of social technology. Understanding the ethical dimension of social technology allows us to identify the role played by a set of laws - Metcalfe's law, law of positive externalities, and law of increasing return to adoption - in the development of social empowerment practices in the aforementioned environments, which are here defined as "communities of knowledge". The ethical vectors present in social technology are analyzed from the following categories: communitarian knowledge and innovation, democracy and citizen participation, dialogue between popular wisdom and scientific knowledge, and holistic educational process.

Key words: social technology, hidden innovation, communities of knowledge, ethics and technology.

* Este artículo ha sido escrito en el marco del proyecto de investigación "Estudio multilineal de las comunidades de conocimiento y acción en el ciberespacio". Plan Nacional de I+D B 09. Proyecto FFI2009-07709. Investigador principal: Ramón Queraltó Moreno. 


\section{Definiciones de innovación oculta y tecnología social}

La innovación oculta está formada por actividades de innovación que no se reflejan en los indicadores tradicionales tales como inversiones en $\mathrm{I}+\mathrm{D}$ en sentido formal o patentes concedidas (NESTA, 2007, 4). No toda innovación está basada en el conocimiento científico, en el I+D tradicional. De hecho, el informe NESTA estima que solo un 7\% de las innovaciones producidas en el Reino Unido entran dentro de esta categoría. El resto, en sentido estricto, formaría parte de la innovación oculta. La innovación social es un tipo particular de innovación oculta. Los parámetros generales para una discusión sobre la innovación social están bien mapeados en una serie de estudios publicados recientemente en castellano.(Echeverría, 2010; Gurrutxaga, 2010; Gurrutxaga y Echeverría, 2010). La Young Foundation define la innovación social como "nuevas ideas que trabajan en pro de la satisfacción de necesidades apremiantes y de la mejora de la vida de las personas" (Mulgan, 2006a, 10). Mulgan defiende que muchos de los elementos que constituyen la vida cotidiana de la sociedad occidental contemporánea fueron en su día innovaciones radicales: vehículos conducidos a gran velocidad por sus propietarios, servicios nacionales de salud, guarderías infantiles, etc. Además, fue la sociedad civil el motor de muchas innovaciones sociales en la historia reciente: asociaciones de ayuda mutua, microcréditos, cooperativas, sociedades filantrópicas, etc. (Mulgan, 2006b, 145).

No hay duda de que el ritmo de innovación a partir de tecnologías sociales se incrementará en los próximos años, por varias razones. La primera, la incapacidad de los gobiernos para dar soluciones a las necesidades crecientes de una población más interconectada, que demanda más calidad de vida, más transparencia en los entresijos de la vida política (véase el caso Wikileaks) y una participación más directa en los asuntos públicos. En segundo lugar, la falta de voluntad, de independencia o de capacidad por parte de dichos gobiernos para oponerse al dominio de los mercados y la lógica neoliberal impuesta por un pensamiento dominante que hunde sus raíces en la ideología de Milton Friedman. En tercer lugar, el empoderamiento de la sociedad civil a partir del uso no oficial e inesperado de todo un arsenal de tecnologías que, por una vez en la historia, alteran el balance de poder entre ciudadano e instituciones a favor de los primeros. En cuarto lugar, la transmisión de valores a través de las herramientas de groupware y comunicación social que caracterizan las redes sociales y otros fenómenos propios de la Web 2.0. Las nuevas reglas del juego político que crean estos valores compartidos se mezclan con experimentos de democracia participativa y metodologías de acción directa, dando lugar a la acción sin precedentes de smart mobs (muchedumbres inteligentes) y flash mobs (muchedumbres relámpago) que comienzan a poner en cuestión los lí- 
mites establecidos de conceptos clave de nuestra filosofía política como democracia, participación y ciudadanía. Sin duda la mezcla de estos cuatro factores, junto con el fracaso de las estructuras socioeconómicas vigentes, dará lugar a una necesaria explosión de innovación social que probablemente dejará pequeñas las transformaciones sociales que ha señalado Mulgan anteriormente.

\section{Tecnología social frente al paradigma neoliberal de la innovación}

La innovación social se ha entendido tradicionalmente como una empresa colectiva de la sociedad, en la cual las estrategias y los lazos comunitarios jugaban un papel fundamental. Un modelo de innovación caracterizado por la autonomía en la definición de las necesidades sociales a las que dicha innovación debía dar respuesta. Sin embargo, el paradigma neoliberal ha conseguido cooptar dicho concepto asimilándolo a un nuevo imaginario social en el que la noción de innovación es una característica de la acción individual de los componentes del colectivo. Son los discursos que relacionan innovación con la gestión (management) en cualquiera de sus formas. La innovación pasa así de ser un asunto de dimensión comunitaria a un problema de gestión individual cuyas claves no son diferentes de aquellas que explican el éxito y desarrollo personales. Una gestión que se aplica con especial énfasis a un uso científico y sistemático de los elementos técnicos que confluyen en el proceso de innovación. No en balde A. N. Whitehead (1926) afirmó que la invención más importante del siglo XIX fue "la invención del método de inventar". Es decir, la innovación dejó de ser algo casual para convertirse en una empresa sistemática, sometida a reglas y a la lógica del método científico.

La innovación tiene una importancia estratégica para el sostenimiento de una sociedad basada en la expansión de la globalización a todos los niveles y en la asociación del cambio social con el cambio tecnológico. Aunque son varias las formas de entender cómo interactúan a nivel causal la tecnología y la sociedad, se han impuesto de facto las teorías del determinismo tecnológico, que colocan al cambio social como una variable dependiente de la evolución tecnológica, con todo el discurso ideológico que dicha afirmación trae consigo. Aunque la etiqueta de determinista tecnológico no es bien recibida por casi ninguno de sus proponentes, no es difícil identificar rasgos de dicha creencia en pensadores como Daniel Bell en El advenimiento de la sociedad postindustrial o Alvin Toffler en El shock del futuro y La tercera ola. Más notable será el caso de Lynn White, el famoso historiador medievalista que hacía de la invención del estribo un elemento determinante para la llegada de la Edad Media y su organización económica y social: el estribo cambió según él el arte 
de la guerra en la Edad Media y permitió montar a caballo con armadura y las manos liberadas para portar armas ofensivas o defensivas. Con el caballero nacieron las órdenes de caballería, y con ellas el sistema feudal.

Nadie duda hoy en día del carácter reduccionista de un determinismo tecnológico fuerte, que hace de la tecnología una variable independiente y causa eficiente del cambio social, pero la creencia en el papel determinante de la innovación para el bienestar social, y la identificación de la innovación con el cambio tecnológico, se constituyen en un pensamiento (casi) único que reduce el universo de discurso y limita el ámbito de acción de una sociedad que necesita algo más que formas más rápidas y eficientes de realizar las mismas tareas sin un cuestionamiento real de los objetivos a los que dichas tareas sirven, sin emprender una necesaria reingeniería de los procesos. Mi tarea en este artículo es mostrar cómo la epistemología vigente de la innovación tecnológica y social no es independiente de una visión ideológica basada en un conjunto de presupuestos éticos que no se hacen visibles en el discurso dominante. Por tanto, para extender la noción de innovación al terreno de lo social desde una perspectiva diferente es necesario mostrar la importancia de una dimensión ética que se manifiesta en el papel que ciertos vectores éticos juegan en el desarrollo de prácticas de innovación que actúan como motores de empoderamiento social y convierten a las comunidades que las utilizan en comunidades de conocimiento, es decir, colectivos que saben utilizar el conocimiento como recurso estratégico para el cumplimiento de fines autodefinidos. Hasta hace poco tiempo se consideraba que solo las empresas y las instituciones eran capaces de usar el conocimiento práctico (knowhow) en estos términos. Por ello la innovación se estudia habitualmente en tres niveles: público-institucional, privado-empresarial e individual.

Alonso y Fernández (2011) mapean con gran exactitud una situación basada en el éxito del discurso apologético de una globalización imparable e incuestionable en sus beneficios, que trajo consigo una reconceptualización de la innovación como una poderosa arma en la búsqueda de ventajas competitivas en todos los terrenos, desde el tecnológico hasta el social, en un contexto de lucha por la apropiación de los mercados. Por tanto, la tecnología social ha pasado a formar parte de un nuevo imaginario social en el que no tiene ya una relación intrínseca con la reclamación de nuevos derechos o la apelación a formas más perfectas de democracia. Por el contrario, su significado pasó a identificarse con la capacidad de competir en un entorno globalizado a través de la adaptabilidad de las sociedades y de su capacidad de mutación para adaptarse a una estructura de mercado en la que poder competir en un plano empresarial a través de la producción de bienes y servicios propios tanto de un capitalismo mercantil como de un capitalismo cognitivo. También señalan varias influencias en este proceso, como la del economista austro-estadounidense Joseph A. 
Schumpeter, los sociólogos de la sociedad post-industrial (Bell), la sociedad de la información y del conocimiento, desde sus vertientes más sociológicas (A. Touraine y M. Castells) hasta las místicas o futuristas (Y. Masuda, A. Toffler), y la de los gurús de la gestión del conocimiento como Nonaka y Takeuchi (1995), que retoman los conceptos de conocimiento tácito y explícito para sugerir cómo las empresas debían generar innovación a partir de procesos de conversión de dichas modalidades de conocimiento (socialización, externalización, internalización, combinación). Otros autores, como Debra Amidon, hacen hincapié en el papel que juega la gestión de las ideas desde su creación hasta el punto de máxima utilización, buscando en este proceso las claves para entender las dinámicas de innovación en las organizaciones (Amidon, 2003).

También defienden Alonso y Fernández (2011) que la innovación se ha alejado cada vez más de utopías sociales o de proyectos que pongan en tela de juicio los valores vigentes de la sociedad occidental, identificándose con productos emblemáticos (tabletas, smartphones, lectores de libros electrónicos, etc.), con nuevos emporios empresariales que venden modelos de vida más que productos (Google, Apple), con empresas fruto de estrategias nacionales de innovación (Nokia, Ericsson) o con herramientas de groupware que transforman de manera significativa las formas vigentes de comunicación social y se convierten en iconos de una nueva juventud (Facebook, Orkut, Twitter, etc.). Creo que en este punto es importante distinguir entre niveles de visibilidad de la innovación relacionada con los citados proyectos. Quizá sean los más visibles porque justifican el statu quo y su valor se refleja en términos financieros y bursátiles. Sin embargo, hay toda una constelación de proyectos de innovación social oculta que no tienen esa caja de resonancia ofrecida por el sistema, y cuyo valor no se cuantifica a través de los indicadores convencionales, pero que afectan a la vida de las comunidades que los promueven y marcan un antes y un después en el equilibrio de poder entre los poderes público y privado, por un lado, y el tercer sector por otro. A la vista de las experiencias exitosas y de alta visibilidad, numerosos estudios se han dedicado a enumerar los factores clave y las condiciones de partida que han posibilitado el éxito de tales experiencias, generando doctrina acerca del camino a seguir para desarrollar culturas de innovación en otras sociedades que permitan su inserción en el mercado globalizado.

\section{La innovación debida al lead user}

Un paso intermedio hacia la identificación de esos vectores éticos relevantes para la innovación es el estudio de los postulados de E. von Hippel (1986). Sus aportaciones sobre la innovación abierta (open innovation) se basan en su con- 
cepto de innovación debida al usuario (user innovation), según el cual una parte importante de la innovación en su conjunto no se debe a los fabricantes de productos sino a los usuarios finales de los mismos. Sus observaciones demostraron que los productos están diseñados habitualmente para cubrir las necesidades genéricas de grandes grupos de usuarios. Pero existen usuarios que tienen necesidades particulares no contempladas por dichos productos genéricos, y sus ideas realimentan el proceso productivo para desarrollar no solamente nuevos artefactos, sino también nuevos servicios o metodologías. Esto lleva al concepto de lead user (usuario innovador o pionero) como figura clave para descubrir necesidades latentes de los usuarios y proponer modelos de resolución de las mismas. En muchos casos, ellos mismos crean el producto o servicio que necesitan. Se convierten así en elementos claves para que las empresas identifiquen las tendencias más radicalmente novedosas y detecten necesidades que serán compartidas en un futuro próximo por un gran número de usuarios. La innovación debida a estos usuarios pioneros no solo es relevante en áreas relacionadas con el consumo doméstico. También destacó su relevancia en el campo de la instrumentación científica, así como en el desarrollo del software libre o de código abierto (open source software). En 2010 publicó un estudio de campo sobre el papel innovador de los usuarios para resolver sus necesidades domésticas, en el que demostró que los consumidores eran responsables de dos a tres veces más de innovación que el sector industrial (von Hippel, 2010).

La figura del lead user trae consigo de forma inevitable desde su propia definición el concurso de un vector ético. En palabras de von Hippel (1986), imaginarse el futuro es difícil para una empresa, pero entenderlo es fácil para el que vive allí. Es decir, el lead user detecta con antelación carencias que percibe en el entorno social en el que se encuentra. Además, espera beneficiarse de forma significativa por el hecho de encontrar una solución a dicha carencia. Por tanto, acaba desarrollando nuevos proyectos o servicios porque el coste (moral, social, económico, etc.) de soportar dicha carencia es mayor que el esfuerzo que supone encontrar una solución. Otras veces, desarrollan el producto o servicio de forma autónoma simplemente no quieren o no pueden esperar a que esté disponible desde el mercado. En cualquier caso, su trabajo tiene un origen social, ya que comparte contexto y necesidades con otros usuarios que se encuentran o se encontrarán próximamente en sus propias circunstancias. De hecho, el concepto de necesidad tiene un carácter social e implica un vector ético de carácter comunitario: Solo se percibe como carencia lo que se percibe como problema, lo que implica una lógica comunitaria en muchos sentidos. Por otro lado, la necesidad es social porque pone en juego recursos cognitivos extraídos de la propia comunidad a la que pertenece, y frecuentemente utiliza 
estrategias colaborativas en tal empeño. Finalmente, el vector ético comunitario aparece también en los fines, ya que el resultado del proceso de innovación no debería ser una solución a la búsqueda de una necesidad artificialmente creada por el sistema, sino que va a tener un engarce social con los problemas autodefinidos por uno o varios miembros de la comunidad.

Suponer que el lead user es un individuo aislado cuyos procesos de pensamiento se explican únicamente a partir de una lógica individual es desconocer las aportaciones de autores que han recibido la denominación de comunitaristas, como Michael Sandel, Alaisdair MacIntyre y Charles Taylor. Llevando la perspectiva de Sandel al terreno de la innovación y la satisfacción de necesidades, podemos decir que el usuario no percibe sus carencias o toma sus decisiones acerca de ellas de forma abstracta o desencarnada, ajeno a su a su entorno social y desvinculado de compromisos normativos con respecto a diversas concepciones del bien o a distintas opciones tecnológicas. El lead user parte necesariamente de una idea de bien, entendido como buena vida en su versión más abstracta o, en términos más concretos, de lo que considera un buen funcionamiento de un producto o servicio. También debe partir de un estado de cosas en el que pueda realizar con mayor eficiencia tareas actuales, o tareas futuras que ahora no puede llevar a cabo. En cualquier caso, la identidad del lead user no es separable de su propia idea de bien. Esta idea se va transformando a través de las experiencias de aquél, del cumplimiento o no de sus expectativas, así como de las opciones tecnológicas disponibles. No parece factible objetivarla alejándose de ella y adoptando un punto de vista imparcial e intersubjetivo que le permita decidir neutralmente sobre nociones de bien alternativas. Tales nociones están esencialmente imbricadas tanto con nuestra identidad individual como con los modelos de vida comunitarios que compartimos, en los que nos construimos como personas a través de procesos de socialización. De esta forma, una aproximación comunitarista enfatiza una dimensión sustancial y situada del bien que tiene sus raíces en la necesidad del vínculo comunitario gracias al cual existimos como individuos. Se opone así a una concepción abstracta y aparentemente neutra del bien, propia del individualismo neoliberal. Frente a este individualismo atomizador, se contrapone una conciencia colectiva que afirma el carácter constitutivo del vínculo social y un entramado normativo inspirado no basado en una neutralidad neoliberal, sino en una concepción sustantiva del bien común (Petrucciani, 2008, 223-4).

Este vector ético comunitarista permite que soluciones sub-óptimas sean adoptadas por una comunidad por encima de opciones más eficaces o eficientes, cuando se adaptan mejor a valores compartidos en una comunidad, cuando son coherentes con una idea de bien sustentada por los miembros de dicha comunidad, y cuando su adopción implica una serie de prácticas y procesos compatibles 
con los valores antes reseñados. En términos prácticos, esta inserción del lead user como expresión de los valores y la idea de bien de su comunidad está presente en muchos de los ejemplos de innovación social más característicos. Entre ellos, el desarrollo del software libre y, más concretamente, la distribución Ubuntu del sistema operativo Linux, que adopta como nombre una regla ética sudafricana que expresa en un lema simple y contundente (yo soy lo que soy por lo que todos somos) el espíritu de cooperación que ha inspirado su desarrollo.

Ubuntu es un concepto tradicional de África del Sur, proveniente de las lenguas zulú y xhosa. Tiene varios significados, pero todos ellos hacen referencia a la dimensión comunitaria de la vida humana, abriendo un camino que une la felicidad personal con la mejora de la comunidad: "Soy porque nosotros somos", "una persona se hace humana a través de las otras personas", "una persona es persona en razón de las otras personas", "yo soy lo que soy por lo que todos somos", etc. Su origen está en la antigua frase zulú "umuntu, nigumuntu, nagamuntu", que significa "una persona es una persona a causa de los demás". (Wikipedia, "Ubuntu", 2012). En la base de este concepto está la creencia de que ser humano es afirmar la humanidad de los demás y establecer sobre este principio relaciones humanas respetuosas. También refleja dos principios básicos de la filosofía tradicional africana: la preservación de la vida como primera prioridad, por encima de la riqueza o el beneficio personal, y el poder real entendido como la consecuencia natural de una cesión voluntaria de todas las personas situadas por debajo del soberano. Por último, esa dimensión comunitaria de Ubuntu se muestra en la costumbre de llamar y referirse a las personas por su apellido más que por su nombre de pila. Con ello de desindividualiza a la persona para asociarla a su linaje, a la familia o tribu de la que proviene. Las consecuencias éticas son claras: uno se convierte en embajador de su propia estirpe, y es así responsable de sus actos en nombre de su familia. Su buen o mal nombre afectará a todos los suyos, los que le han precedido y los que le seguirán. De esta manera se crea una apelación a la dignidad y la lealtad muy superior a la que nuestras escuelas enseñan. Es una ética no solo de las generaciones futuras, sino también de las pasadas; es una ética de la vida distinta de la que propugna el evolucionismo en economía política, como veremos a continuación.

\section{Sobre la teoría evolucionista en la economía politica}

El evolucionismo económico parte de la obra de Nelson y Winter An evolutionary theory of change (1982), y explica los comportamientos de los agentes como consecuencia de dos factores: las rutinas heredadas y los mecanismos de búsqueda como consecuencia de un entorno cambiante. De esta forma se ex- 
plica el proceso de decisiones en torno a la innovación haciendo hincapié en los mecanismos cognitivos que conforman las representaciones del mundo para un sujeto, y el hecho de que la definición de fines a alcanzar y de medios instrumentales escogidos para ello se construyen en función de dinámicas de aprendizaje y socialización, lo que introduce una racionalidad procedimental frente a la racionalidad paramétrica del modelo clásico. Se mantiene la creencia schumpeteriana en el papel de la innovación como motor del cambio social y el crecimiento económico, pero se coloca en el centro a la empresa y no al individuo innovador, ya que se considera que solamente la empresa es el lugar adecuado para el desarrollo de una producción a la vez creativa y altamente socializada. Solo la empresa poseería las estructuras con la articulación y coherencia necesarias para transformar experiencias en aprendizajes interactivos, acumulando así los conocimientos ligados a la innovación y la producción.

Los usuarios tienen también un papel importante, pero en cierta forma periférico: su acción es relevante porque se sitúa en el ecosistema de la empresa. Los procesos de uso, de imitación y de difusión son creadores, a la manera de von Hippel, y el beneficio dependerá de los rendimientos crecientes de los usos innovadores más que de los rendimientos crecientes de adopción. La propia heterogeneidad creciente del medio social y económico permitirá la supervivencia de aquellas empresas que mejor se adapten a través de nuevas rutinas generadas como respuesta a un entorno de fuerte competencia. Esta teoría evolutiva somete la innovación a los dictados de la lucha por la supervivencia y de la selección natural, en lugar de confiar en las dinámicas sinérgicas o las externalidades positivas. Innovar no es para el evolucionismo económico-político una actividad con resonancias éticas, sino más bien una obligación ontológica. Una resistencia frente a la angustia que genera un mundo en el que la necesidad y la escasez son las reglas de juego que definen el entorno. Por tanto, esta teoría aporta una visión de la riqueza menos ligada a elementos materiales y de cooperación (restringida a la empresa) como factor esencial para la innovación, además de desplazar la racionalidad paramétrica propia de un sujeto por la racionalidad procedimental de actores múltiples que maximizan la utilidad esperada de las consecuencias de sus decisiones en un entorno en el conviven fuerzas cooperativas y lucha por recursos escasos cuya apropiación garantiza la supervivencia.

Se echan de menos en esta teoría vectores éticos que permitan tres avances. En primer lugar, un vector que ponga en valor la riqueza social acumulada y el placer que los agentes obtienen al aumentarla y compartirla. En segundo lugar, otro vector que permita extraer las máximas posibilidades de las herramientas de cooperación que la tecnología está poniendo en las manos de las personas. En tercer lugar, el reconocimiento de que la riqueza oculta del nuevo entorno de la 
economía del conocimiento tiene menos que ver con el dinero, con los bienes tangibles que conservan su valor o lo incrementan con el paso del tiempo, y más con los tomates y las lechugas, con los bienes perecederos que uno pueda encontrar en un supermercado; bienes con fecha de caducidad, que van perdiendo su valor a medida que desciende su frecuencia de uso o la utilidad asignada por los usuarios; bienes que no permiten su acumulación indefinida ni su conservación más allá de las transformaciones evolutivas de las necesidades y expectativas de los agentes que intervienen en este ecosistema. En cuarto lugar, las leyes de la evolución explican con más facilidad la destrucción que la creación, la extinción de especies digitales más que la aparición de otras nuevas. ¿No ha llegado la hora de poner en tela de juicio la lógica de la competencia y la lucha por la supervivencia frente a la sinergia y la búsqueda convergente de objetivos compartidos más allá de una lógica de juegos de suma cero o de conflicto puro? Parece que el problema consiste en no aceptar los riesgos de una cooperación libre y abierta, que hiciera de la compartición, según lo expresa el viejo adagio todo lo que no se da, se pierde, su leitmotiv (Corsani, 2004).

\section{Vectores éticos en tecnología social}

La tesis que propongo en este artículo es que parte de dichas transformaciones producidas en el ámbito de la tecnología social se manifiestan en forma de innovación oculta, que solo puede aflorar y difundirse a partir de una serie de vectores éticos que caracterizan la acción de comunidades que pueden calificarse de entornos colaborativos. Esta acción se concreta en forma de prácticas que a su vez se convierten en herramientas de empoderamiento social. Cuando eso ocurre en cierto grado, podemos hablar de dichos entornos colaborativos en términos de comunidades de conocimiento y acción. Para poder identificar de manera adecuada lo que puede ser considerado tecnología social en dichos entornos es necesario que la participación de los usuarios y beneficiarios de la tecnología participen en todas las etapas de análisis del problema y construcción de soluciones a los mismos. No es suficiente que se desarrolle una solución técnica adecuada y se transfiera a la comunidad, pues ello compromete el proceso de empoderamiento social. Es necesaria la presencia de una serie de vectores éticos que garanticen el compromiso, el aprendizaje y la emancipación social, promoviendo así factores intangibles que van más allá del simple desarrollo de artefactos:

1. Se entenderá la innovación social como un conjunto de técnicas y metodologías transformadoras desarrolladas o aplicadas en interacción con la población y apropiadas por ella, que representan soluciones para la inclusión social y la mejora de las condiciones de vida de la comunidad. 
2. Sus principios deben promover la inclusión de los usuarios en todas las etapas de del desarrollo del producto, servicio o metodología.

3. Aprendizaje y participación son procesos que deben caminar juntos para hacer de la innovación una práctica de empoderamiento social.

4. La transformación social producida por dicho proceso de innovación debe comprender la realidad de manera sistémica. No puede reducirse a ser la solución particular a un problema restringido sin referencia a una praxis transformadora de la realidad que ofrezca pistas para la resolución de otras carencias sociales relacionadas con la anterior.

5. Todo miembro de la comunidad debe ser capaz de generar conocimiento y de aprender y enriquecerse a partir de este proceso.

A través de estos vectores éticos el proceso de innovación social une a la excelencia técnica de la innovación (eficiencia y sostenibilidad), tres dimensiones que inciden sobre su relevancia social, haciendo que los miembros de la comunidad sean sujetos activos en un proceso democrático participativo y no solo receptores en un proceso de transferencia de tecnologías. Estas dimensiones son las siguientes:

1. Conocimiento e innovación comunitarios: el proceso de innovación social debe comenzar a partir del reconocimiento de las problemáticas sociales, debe llevarse a cabo a través de pautas de organización y sistematización, y como resultado generará innovación dentro de las propias comunidades.

2. Participación, ciudadanía y democracia: desde su comienzo este proceso debe promover una ampliación de la democracia y la ciudadanía, debe utilizar metodologías participativas y buscar la inclusión social y la accesibilidad, de forma que alcance a un número máximo de personas y no discrimine ni a portadores de necesidades especiales ni a personas caracterizadas por su diversidad funcional. Rescate de la autoestima, revalorización de ,os saberes locales e afirmación identitaria se convierten aquí en pilares de desarrollo.

3. Educación: el proceso de innovación social debe ser considerado desde el punto de vista de la educación como un proceso pedagógico holístico, debe dar lugar a un diálogo continuo y sistemático entre saberes populares y saberes científicos, para posibilitar su apropiación por parte de las comunidades, de forma que ganen autonomía. Este vector entronca con uno de los postulados de las teorías evolucionistas del cambio tecnológico, que conciben la innovación como un proceso creativo de aprendizaje social. (ITS, 2004 y 2007). 
La forma en que se potencian estos vectores éticos de la tecnología social viene a través de la aplicación de tres leyes que permiten completar el nexo de unión entre tecnología social e innovación oculta, permitiendo así poner en evidencia factores que suelen pasar por alto los indicadores convencionales de innovación formal: la ley de Metcalfe, la ley de externalidades positivas y la ley de rendimientos crecientes de adopción.

La ley de Metcalfe, creador de la topología de red Ethernet, tiene una relevancia fundamental en teoría de redes y afirma que en el valor de una red de comunicaciones aumenta proporcionalmente al cuadrado del número de usuarios del sistema. Es especialmente útil para explicar muchas de las novedades que trae Internet o las redes sociales en relación a otros sistemas colaborativos clásicos. Desde su formulación en 1976 ha habido opiniones discrepantes sobre la función matemática que expresa su crecimiento exacto, aunque no sobre el fondo de la cuestión: en entornos colaborativos, ya sea una red social, un sistema de computación distribuida o un sistema de comunicaciones, el valor de la red aumenta cuantos más miembros tenga. Para B. Briscoe, A. Odlyzko y B. Tilly (2006), Metcalfe sobreestima el valor de los nodos de la red al suponer que todos tienen una capacidad homogénea de comunicación -- es decir, un número similar de conexiones potenciales con otros nodos - y por tanto defienden que es exagerado hablar de una función cuadrática al hablar del crecimiento del valor de la red, decantándose por un crecimiento logarítmico: el valor de una red de n nodos no aumenta con el cuadrado de n, sino n veces el logaritmo de n. En sentido opuesto, David P. Reed propone que la utilidad en las grandes redes, especialmente las redes sociales, crece exponencialmente con el tamaño de la red, ya que el número de subconjuntos posibles de miembros de la red aumenta mucho más rápidamente que el número de usuarios o de conexiones entre ellos. Estos subconjuntos añaden valor a la red por encima de los usuarios considerados individualmente, y por ello existe un componente de cooperación que excede los cálculos de Metcalfe.

La relevancia de la ley de Metcalfe para la consideración de los vectores éticos en tecnología social proviene del hecho de poder aplicarse a cualquier sistema en que el intercambio de información entre sus elementos juegue un papel clave, y éste es precisamente el caso de los sistemas de tecnología social. Además, otro factor justifica su inclusión en este elenco de vectores éticos. El valor económico de los bienes tangibles está sometido a la ley de la oferta y la demanda. Su precio está en función de su escasez y de la demanda de los mismos. Cuanto más escaso sea un bien y más buscado sea, mayor valor de intercambio posee. Como consecuencia, una sobreabundancia de dicho bien tiene consecuencias inflacionistas, pues pierde valor a me- 
dida que oferta y demanda tienden a equilibrarse. Metcalfe lo sabía bien al poner el ejemplo del fax. Cuando solo una persona posee tal aparato, su valor es mínimo al no tener con quien comunicarse. Cuantas más personas tengan un fax, más valor tendrá para cada uno de ellos al multiplicarse las posibilidades de comunicación con otros nodos de la red. Se abre así una nueva economía en la que la distribución de la riqueza no responde al modelo formal de los juegos de suma cero, y donde el beneficio de los demás agentes redunda en el nuestro propio, quebrando así uno de los paradigmas de los modelos evolutivos de la economía política, basados en la competición y la innovación como búsqueda de factores diferenciales que maximicen las posibilidades de supervivencia en un entorno que tiene la escasez de recursos como punto de partida. También se resuelven algunos de los problemas que genera un modelo de tipo tragedia de los comunes - según fue enunciado por Garret S. Harding - en el que el sumatorio de las ventajas individuales acaba siendo nefasto para la colectividad. Lo que está aquí en juego no es ya un problemas de topología de redes, sino de paradigmas éticos que pueden justificar a través de la teoría de juegos las ventajas de la cooperación. E1 caso del software libre es un buen ejemplo de ello.

La segunda es la ley de externalidades positivas, de Alfred Marshall y Arthur Pigou. Cuando una operación económica entre dos agentes tiene efectos sobre un tercero sin que haya intercambio entre alguno entre aquéllos y éste, se crea una externalidad ${ }^{1}$. Si la externalidad opera en detrimento del tercer agente, se trata de una externalidad negativa o de una deseconomía externa. Si el tercer agente ve mejorar su posición, aumentar su bienestar, su beneficio, sus posibilidades de acción o sus activos inmateriales, se dice que se ha creado una externalidad positiva. A. Marshall introdujo en 1920 la noción de externalidades tecnológicas positivas con el ejemplo de una empresa que por su implantación geográfica beneficia al área en que se instala más allá de su contribución fiscal, mercantil o productiva. Por ejemplo, creando una demanda de mano de obra de alta cualificación que incentiva otras empresas a instalarse y mejorando el sistema educativo a través de la creación de nuevos planes de estudios y la posibilidad de contratar especialistas como profesores. Arthur C. Pigou (sucesor de Marshall en Cambridge y predecesor de J. M. Keynes) de-

\footnotetext{
${ }^{1}$ Se denomina beneficio privado al que obtiene el consumidor de un bien o el usuario de un servicio. El beneficio externo será el obtenido por otros consumidores o usuarios por causa de la operación anterior. En cada caso, se añade el apelativo marginal a cualquiera de los beneficios anteriores cuando se consume una unidad más del bien o servicio. El beneficio social marginal será la suma del beneficio marginal privado y externo de todos los individuos.
} 
fendió que las políticas de inversión pública crean externalidades positivas que permiten distinguir entre la utilidad social y la simple suma de utilidades individuales de los agentes implicados. Un ejemplo de transferencia pública de este tipo es el caso de la Ruta 128 en Boston.

La Ruta 128 (Route 128 o Yankee Division Highway) es una autopista de circunvalación en forma de semianillo de unos 20 kilómetros de radio que rodea la ciudad de Boston. Desde los años 60 hasta los 80 se produjo un gran incremento del número de industrias de alta tecnología instaladas en las áreas suburbanas a lo largo de la Ruta 128, y pasó a convertirse en un símbolo del Boston tecnológico. Ha sido el polo más importante en I+D en Estados Unidos después del Silicon Valley a finales de los años 80, con más de 1200 empresas entre las que se incluyen Digital Equipment, Microsoft, Data General, Computervision, Polaroid, Sun Microsystems, Autodesk, Raytheon y otras. Ha sido conocida popularmente como America's Technology Highway, aunque nunca se aceptó el cambio oficial de nombre, y su construcción es considerada como el factor desencadenante de lo que se llamó el milagro económico de Massachusetts. Proyectada inicialmente en 1912, fue calificada como una autopista a ninguna parte" ya que no conectaba grandes núcleos urbanos entre sí, sino que unía áreas suburbanas próximas a carreteras radiales que salían del centro de Boston, evitando entrar en el área metropolitana. Entre los años 1936 y 1941 fue ampliada significativamente en capacidad y longitud. Su financiación fue fundamentalmente pública. Fue completada en 1959 con un coste total de 63 millones de dólares de la época, aunque posteriormente tuvo que ser modificada en varias ocasiones para poder absorber la explosión de tráfico que se produjo como consecuencia del boom económico.

Ya en 1948 varios estudios destacaron que su proximidad al Instituto de Tecnología de Massachusetts (MIT) sería un factor clave para el desarrollo de una fuerte industria local de altas tecnología. Las externalidades positivas estaban presentes en los modelos que los planificadores urbanos aplicaron a la región. Al proyectar la Ruta 128 en terrenos no urbanizados más allá de las áreas desarrolladas se circunvalaban los centros urbanos, se mantenía un bajo costo de los terrenos y se evitaban problemas con los habitantes de áreas residenciales. Las autopistas comunicaban estos terrenos con una mano de obra especializada y con los investigadores del MIT y de otros universidades del área metropolitana de Boston. A la vez se favorecía la creación de parques industriales fuera de la típica estructura de la industria tradicional, caracterizada por su concentración y centralización, pasando a construcciones de baja altura, separadas por extensas áreas verdes, con aparcamientos al lado de cada edificio, facilitando la obtención de permisos urbanísticos, la calidad en las 
condiciones de trabajo y la expansión de las empresas. Desde 1953 hasta 1961, 169 empresas con más 24000 empleados se instalaron a lo largo de la Ruta 128. En los años cincuenta muchas de las poblaciones en torno a la autopista cuadriplicaron su población, doblándola de nuevo a lo largo de la década de los 60. Hoy en día tiene la denominación oficial I-95 e I-93, aunque para muchos de los residentes en el área seguirá siendo la Ruta 128.

Este caso de la Ruta 128 es un claro ejemplo de economía externa positiva. Los parques tecnológicos muestran cómo la creación de infraestructuras comunes incentiva la implantación de nuevas empresas y beneficia a otros agentes que no participan de los gastos de inversión. Las externalidades permiten abordar los problemas de intercambio y de coordinación en sistemas complejos en los que no existe una contrapartida monetaria regulada por un sistema de mercado (Moulier, 2004, 147-8), como es el caso de la tecnología social. Esta ley nos muestra por un lado la transmisión osmótica de conocimiento que se produce entre agentes de un sistema de tecnología social, y por otro el beneficio para terceros que no participan directamente en el desarrollo de una innovación social, como es el caso del software libre y también el de la pacificación de las favelas en Rio de Janeiro².

Para explicar esta transmisión de conocimiento en un sistema de tecnología social será necesario introducir la noción de óptimo de Pareto. Es un concepto de la investigación operativa y la teoría de juegos que señala el punto máximo de beneficio que puede alcanzar un agente sin perjudicar a otros. En la búsqueda del beneficio colectivo, los agentes pueden seguir intentando maximizar la utilidad esperada de sus decisiones hasta un punto en el que en los intercambios perjudiquen a algunas de las partes. Ese sería el límite de la riqueza común, el punto en el que nadie mejorar su posición en el juego sin perjudicar a otra persona. Nos permite establecer tanto negociaciones entre agentes como análisis coste-beneficio aplicables a procesos de innovación. La tecnología social es una situación de optimización multiobjetivo en la que varios factores deben tenerse en cuenta: se busca en muchos

\footnotetext{
${ }^{2}$ Tras permanecer un tiempo en la Favela Cantagalo, en Rio de Janeiro, en 2012, pude comprobar hasta qué punto las favelas cariocas se han convertido en un extraordinario laboratorio de innovación social donde la aplicación del conocimiento periférico y la utilización inteligente de estrategias de integración social provenientes de la sociedad civil están consiguiendo una transformación de enormes dimensiones de áreas de marginalidad que han resistido durante décadas cualquier tipo de política gubernamental de integración social. No es éste el lugar para tratar de este caso en profundidad, pero solo quiero dejar apuntado que una comprensión de las externalidades positivas a la brasileña es un elemento clave en la lucha contra la pobreza, y será un elemento central de la transformación social que experimentará el gigante sudamericano en las próximas décadas.
} 
casos la innovación más eficiente (más eficaz en relación a su costo), por lo que un óptimo de Pareto sería aquella innovación tal que no exista otra más eficaz y más barata. Sin embargo, para que se pueda hablar de tecnología social, deben cumplirse otros requisitos: que esté disponible para los miembros de la comunidad, que su proceso de desarrollo sea social en todas las etapas, que se convierta en proceso holístico de aprendizaje para la comunidad, que aumente su autonomía, etc. En este caso, sería perfectamente posible escoger una innovación sub-óptima que, al ser adoptada por la comunidad, se convierta en la mejor opción disponible. Por otro lado, el concepto de óptimo de Pareto no supone la introducción de ningún vector ético, dado que no prejuzga acerca de las distribuciones de los recursos o los beneficios entre los agentes a partir de una situación de partida determinada, denominada técnicamente dotación inicial. En ese sentido se sitúa la crítica de economistas y teóricos de la justicia como Amartya Sen. Queda también el problema de cómo compensar a aquellos que son perjudicados por una deseconomía externa (externalidad negativa).

La tercera es la ley de rendimientos crecientes de adopción, de Brian Arthur (1989). Aplicada a este contexto, afirmaría que cuanto mayor es el número de participantes en las comunidades virtuales, mayor es la utilidad de la herramienta para cada uno de ellos, y mayor utilidad práctica y relevancia científica tiene la información creada a partir de esa interacción. La ley de rendimientos crecientes es uno de los términos del nuevo paradigma económico derivado de una apropiación social de las TIC que participan de la afirmación de una innovación oculta. El concepto fue acuñado por Brian Arthur (1989) para mostrar el papel de la difusión, adopción e imitación como fuente de valor económico a través de la siguiente tesis: una tecnología no se elige por ser la mejor entre las alternativas disponibles, sino que se convierte en la mejor como consecuencia de haber sido elegida. La argumentación de Arthur se basa en tres elementos. En primer lugar, la disminución de los costes de producción a medida que el producto o servicio es adoptado por más usuarios, de acuerdo a las leyes de la economía de escala. En segundo lugar, por el perfeccionamiento conseguido a través de la experiencia, transformado en know-how por los usuarios. En tercer lugar, aplicando la ley de Metcalfe o la ley de Reed el valor del producto o servicio aumentará en función (cuadrática o exponencial) del número de usuarios, y además dicho valor - en términos de utilidad - beneficiará a otros componentes de la red que no hayan participado en su desarrollo, de acuerdo con la ley de externalidades positivas. Como consecuencia, no es necesario que se imponga un óptimo de Pareto según los términos estrictos de la eficiencia económica, sino que la adopción de una innovación subóptima, a 
partir de una masa crítica de usuarios, se convierte en una opción perfectamente racional ${ }^{3}$.

Estas serían las consecuencias desde un punto de vista economicista centrado en costes de producción y precios de productos y servicios, pero desde un punto de vista ético se pueden apreciar los cambios que la aplicación que esta ley trae a la socialización de los procesos de innovación social en los que el conocimiento periférico cobra un papel clave, tarea que emprende Jollivet (2004) con su crítica a la ley de Arthur y su propuesta de modificación de la misma para poner el acento en la forma en que el proceso de innovación transforma más a la comunidad que al propio producto. Desde este punto de vista, la ley de Arthur se encuentra constreñida a un círculo cerrado de factores económicos: oferta y demanda, producción y consumo, consumo pasivo como acto de destrucción. También el aprendizaje, critica Jollivet, sería una actividad automatizada, en la que el valor del conocimiento adquirido se transforma esencialmente en aumento de productividad. El consumidor sería también una figura pasiva, ya que a través del hecho de usar una nueva tecnología se limitaría a revelar el aumento de utilidad de la misma, sin acentuar los cambios que dicho uso producen en él. Para salir de este impasse es necesario destacar que la utilización es por sí misma creativa, y que los rendimientos crecientes deben serlo de la adopción innovadora, o del uso innovador. Este sería el vector ético final que querría resaltar. La innovación transforma tanto a la comunidad que innova como al producto. Lo mismo ocurre con la adopción de dicha innovación. La innovación no se aliena con respecto de aquellos que la desarrollan: su adopción es socialmente transformadora, puesto que es producción creativa de usos transformadores. El producto o servicio resultado de dicha innovación se transforma a través de su difusión y compartición, a menudo de forma crucial y a veces de forma revolucionaria cuando es socialmente apro-

\footnotetext{
${ }^{3}$ El caso del software libre vuelve a ser la piedra de toque en este punto. Puede que un programa propietario llegue al mercado en una versión más acabada y depurada que su equivalente en código abierto. Sin embargo, el hecho de ser una caja negra para el usuario y estar prohibidas su manipulación, modificación o mejora por parte del usuario, hace que vaya perdiendo terreno frente al programa en código abierto, que se mejora cuanto más se usa, cuantos más usuarios transforman su experiencia en conocimiento compartido. No resulta contraproducente sacar al mercado las versiones beta de un programa de código abierto, ya que así se da la oportunidad de ajustarlo mejor a las necesidades finales de los usuarios. Por otro lado, la adopción de un programa como estándar es crucial para convertirlo en el más útil. La historia de la informática está llena de ejemplos de excelentes sistemas operativos y programas que sucumbieron frente a alternativas menos sofisticadas al no conseguir una masa crítica de usuarios; muchas veces por la terquedad de las empresas de mantener bajo control toda la información técnica del programa y no liberar el código fuente para que otros fabricantes desarrollaran drivers para periféricos o interfases para dicho sistema operativo.
} 
piado para redefinir su relación instrumental con nuevos usos estratégicos autodefinidos por una comunidad.

Por tanto, la adopción no es consumo destructivo sino producción, y producción de usos innovadores. El uso social es por sí mismo innovador, siempre que tengamos criterios que nos permitan ver esta innovación oculta. Para ello debemos partir de unas visión de las redes que vaya más allá de una mera distribución topológica de nodos y enlaces, y rellenar las redes de contenido humano. Así, los vectores de eficiencia económica y los vectores éticos no son realidades disjuntas, ni se puede separar la innovación de una clara visión de las necesidades sociales y los medios lícitos para satisfacerlas. Igual que en las comunidades de conocimiento y software libres, la suma de un miembro-coproductor más a la comunidad de desarrolladores no solo incrementa el valor de uso del contenido de la red, según los dictados de la ley de Metcalfe y la ley de rendimientos crecientes de adopción, sino que mejora, de forma cualitativa, el propio objeto técnico, en beneficio comunitario, a través de la innovación social. La utilización no es consumo destructivo, sino producción innovadora. Cuantos más son los usuarios que se comprometen en una dinámica de adhesión - imitación - adopción, más crece la calidad y el valor de uso del bien en cuestión. Los rendimientos de uso innovadores son radicalmente crecientes. En consecuencia, las externalidades positivas derivadas de un proceso colaborativo de innovación no son una simple combinatoria, sino la expresión de las capacidades de innovación del trabajo cooperativo voluntario que se lleva a cabo en redes de fuerte interacción social. Son expresiones ocultas de la creatividad social (Jollivet, 2004).

Por último, podemos aun dar un paso más allá. Como hemos visto, estamos hablando de procesos que no tienen solo una dimensión económica, sino que conciernen a la socialización de los procesos de innovación. Cuando compartimos productos en red nos vemos obligados a adaptarlos a nuestro propio ecosistema de medios y necesidades. El simple hecho de compartir archivos musicales o vídeos nos obliga a aprender a adaptar su formato y tamaño a los requisitos técnicos de nuestros reproductores: capacidad de memoria, velocidad de reproducción, protocolos soportados, etc. El simple hecho de hacer utilizable en un dispositivo concreto un archivo de uso compartido nos hace desarrollar un nuevo conocimiento sobre formatos de audio y vídeo. Es innovación oculta en tanto que se desarrolla una nueva capacidad personal que realimenta la red a través de los foros de usuarios, y que permite que, al hacer pública la solución de un problema técnico compartido por otros usuarios, éstos liberen su tiempo y su poder mental para dedicarse a resolver otros problemas que acabarán beneficiando a terceros. No es, en ningún caso, una lógica de la eficiencia, sino un paradigma ético basado en un reconocimiento de las posi- 
bilidades innovadoras que la tecnología pone en nuestras manos. La participación en las redes de innovación es, desde este punto de vista, un ejercicio creativo de ciudadanía digital. La adopción de las prácticas de procomún en la red transforma tanto al que las adopta como al medio utilizado, y también a los productos innovadores desarrollados en la red. Nada queda igual que antes. Tampoco los usuarios son los mismos. El factor de contagio a otros campos de la actividad social es evidente: el mismo papel que uno puede jugar al recuperar el control sobre la innovación social puede aplicarse a otros campos del ejercicio de la ciudadanía (Bustamante, 2011). Los rendimientos de uso son, como defiende Jollivet (2004), radicalmente crecientes, pues son la expresión de la capacidad de innovación del trabajo cooperativo voluntario.

\section{BIBLIOGRAFÍA}

Alonso, L. y Fernández, C. (2011): "La innovación social y el nuevo discurso del management: limitaciones y alternativas". Arbor, vol. 187 - 752 nov-dic 2011, pp. 1133-1145.

Amidon, Debra M. (2003): The Innovation Superhighway: Harnessing Intellectual Capitals for Sustainable Collaborative Advantage, Nueva York: Butterworth.

Arthur, B. (1989): “Competing technologies, increasing returns and lock-in by historical events", Economic Journal, vol. 99, num 3, pp 116-131.

Briscoe, B., Odlyzko, A. y Tilly, B. (2006): "Metcalfe's Law is Wrong”, IEEE Spectrum, July 2006, disponible en http://spectrum.iee.org/computing/networks/metcalfes-law-is-wrong, último acceso 20/12/2012.

Bustamante, J. (2011): "Development of a Netlike (Reticular) Ethics as a Paradigm for Digital Citizenship in a Multi-Cultural Society". In NOMADS. Critical Review of Social and Juridical Sciences, vol. 1 no. 2. pp. 31-46. Madrid: Plaza y Valdés

Corsani, A. (2004): "Hacia una renovación de la economía política. Antiguos conceptos e innovación teórica", en Y. Moulier Boutang et al. Capitalismo cognitivo: propiedad intelectual y creación colectiva. Traficantes de sueños: Madrid.

Echeverría, J. (2010): “El debate: Innovación sin ciencia”, Revista Iberoamericana de ciencia tecnología y sociedad. Disponible en: http://www.revistacts.net/index. php?option=com_content\&view=article\&id $=320$ :el-debate-innov.., último acceso 23/12/2012.

Gurrutxaga, A. y Echeverría, J. (2012): La luz de la luciérnaga. Diálogos de innovación social, Madrid: Plaza y Valdés. 
Gurrutxaga, A. (2010): Recorridos por el cambio, la innovación y la incertidumbre, Bilbao: Universidad del País Vasco.

Instituto de Tecnologia Social - Brasil (2007): Para entender a tecnologia social: Uma viagem pelo Brasil. ITS, São Paulo.

Instituto de Tecnologia Social - Brasil (2004): Caderno de debate: direito à ciência e ciência para a cidadania, ITS: São Paulo.

Jollivet, P (2004): “Anexo 2: "Los rendimientos crecientes”, en Y. Moulier Boutang et al. Capitalismo cognitivo: propiedad intelectual y creación colectiva. Traficantes de sueños: Madrid.

Moulier, Y., (2004) "Las externalidades", en Y. Moulier Boutang et al. Capitalismo cognitivo: propiedad intelectual y creación colectiva. Traficantes de sueños: Madrid.

Mulgan, G. (2006a): What's Social Innovation, Young Foundation: Londres.

Mulgan, G. (2006b): “The Process of Social Innovation”, Innovations, Spring 2006, vol. 1, No. 2, MIT Press: Cambridge, MA.

NESTA (2007): Hidden Innovation, Nesta Research Report. London. Disponible en: www.nesta.org.uk/assets/pdf/hidden_innovation_report_NESTA.pdf, último acceso en 20/12/2012.

Nonaka, I. y Takeuchi, H. (1995): The Knowledge Creating Company, Oxford University Press: Oxford.

Petrucciani, S. (2008): Modelos de filosofía política, Buenos Aires: Amorrortu.

Von Hippel, Eric A., Jeroen P. J. de Jong, and Steven Flowers (2010): “Comparing Business and Household Sector Innovation in Consumer Products: Findings from a Representative Study in the UK." SSRN eLibrary, disponible en http://papers.ssrn.com/ sol3/papers.cfm?abstract_id=1683503, último acceso 20/10/2012.

Von Hippel, E. (1986): "Lead Users: A Source of Novel Product Concepts", Management Science, 32(7), 791-805. Disponible en http://web.mit.edu/evhippel/www/papers/evh-01.htm, último acceso 20/10/2012.

Reed, D. P. (1999): “Weapon of Math Destruction”, Context Magazine, Spring 1999.

Ubuntu, entrada en Wikipedia. Disponible en http://es.wikipedia.org/wiki/Ubuntu_\% 28filosof\%C3\%ADa\%29. Último acceso, 28/12/2012).

Whitehead, A. N. (1926): Science and the Modern World. Cambridge University Press: Cambridge. 